\title{
Mcl1 becomes ubiquitin-ous: new opportunities to antagonize a pro-survival protein
}

\author{
Michael D Hogarty ${ }^{1,2}$ \\ ${ }^{1}$ Division of Oncology, The Children's Hospital of Philadelphia; ${ }^{2}$ Department of Pediatrics, The University of Pennsylvania School \\ of Medicine, Colket Translational Research Building, Room 3020, 3501 Civic Center Boulevard, Philadelphia, PA 19104, USA \\ Cell Research (2010) 20:391-393. doi:10.1038/cr.2010.37; published online 1 April 2010
}

Cancer remains a major cause of human mortality. The greatest barrier to cure is resistance to conventional medical treatments. Therapy resistance arises through clonal evolution driven by genomic instability, fostered by selection as therapy-sensitive cells are eradicated. Such resistance has been attributed to alterations in numerous cellular pathways, yet many ultimately impact the process of mitochondrial apoptosis. The critical gatekeepers for mitochondrial apoptosis initiation are the Bcl2-homology (BH) proteins, whose interactions integrate the varied stressors a cell encounters and provide a live-or-die decision (reviewed in [1]). As such, BH proteins are the frequent target of alterations in cancer cells during oncogenesis and tumor progression [2].

Diverse cell stressors, chemotherapy and radiotherapy among them, activate downstream death signaling proteins within the BH3-only family (named for their inclusion of a specific BH death domain). Once activated, these $\mathrm{BH} 3$-only proteins (such as Bid, Bim, and Noxa) are free to interact with multi-domain $\mathrm{BH}$ proteins that reside at the outer mitochondrial membrane. Mitochondrial apoptosis requires a $\mathrm{BH} 3$-only protein to activate pro-apoptotic Bak or Bax, leading to their homo-oligomerization

Correspondence: Michael D Hogarty

E-mail: hogartym@email.chop.edu and release of apoptogenic factors from the mitochondria. This is a "point of no

return" for a cell as it leads to terminal caspase activation as well as bioener-

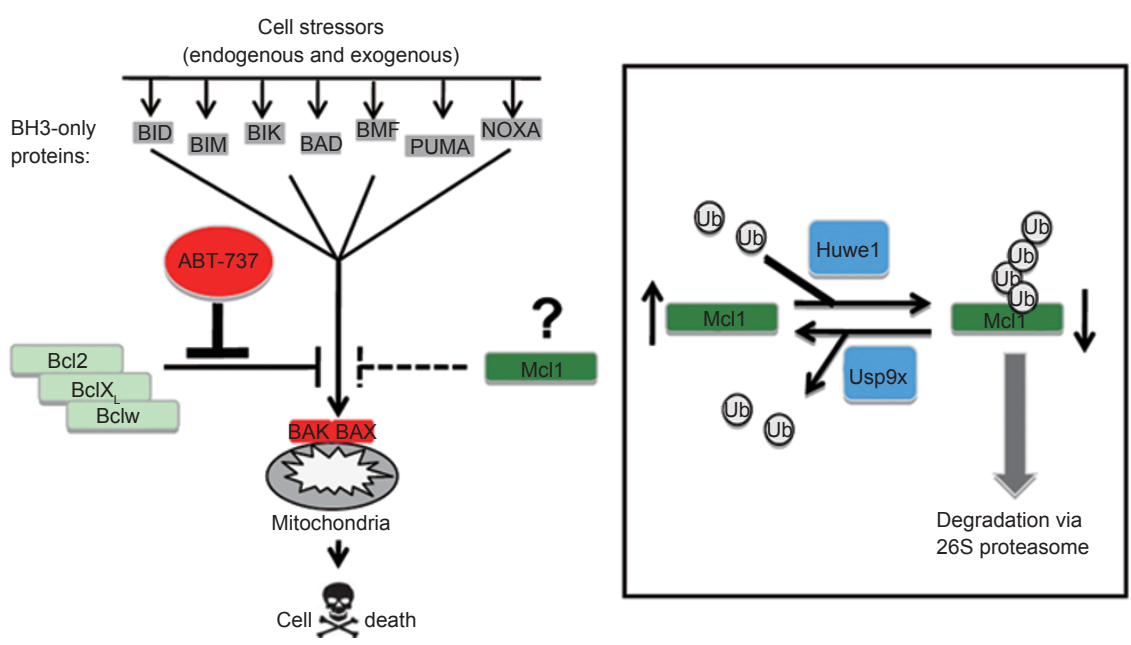

Figure 1 Diverse cellular stressors activate members of a family of BH3-only death-domain containing proteins that can activate mitochondrial apoptosis. This pathway requires activation of the obligate executioner proteins, Bak and Bax. Repressing this process are a family of pro-survival Bcl2-homology $(\mathrm{BH})$ proteins, among them $\mathrm{Bcl} 2$ and $\mathrm{Mcl} 1$. These sequester $\mathrm{BH} 3-o n l y$ proteins preventing apoptosis until the level of activated BH3-only proteins exceeds the cells ability to neutralize them. Cancer cells commonly upregulate these family members to provide a survival bias against cell stressors, including those initiated in response to chemoradiotherapy. Small molecule Bcl2-family antagonists, like ABT-737, can block the pro-survival function of $B c l 2, B c l X_{L}$ and $B c l w$ by competitive displacement. Mcl1 (and A1/Bfl1) are not antagonized by ABT737 so their presence maintains a cancer cell survival bias even in the presence of this drug. Mcl1 itself is regulated by PTMs including phosphorylation downstream of signal transduction pathways (not shown) and the ubiquitinproteasome pathway. Huwe1 is responsible for steady-state destabilization of Mcl1 via ubiquitination and enhanced proteasomal degradation, whereas Usp9x impedes this process through deubiquitination. The balance of these processes may determine the stability of Mcl1 which in turn may impact whether the cancer cell efficiently undergoes mitochondrial apoptosis. 
getic collapse (Figure 1).

An alternative outcome for an activated $\mathrm{BH} 3$-only protein is to be neutralized by a pro-survival $\mathrm{BH}$ protein. These family members (including $\mathrm{Bcl} 2, \mathrm{BclX}_{\mathrm{L}}, \mathrm{Bclw}, \mathrm{Mcl1}, \mathrm{BclB}$ and A1/Bfl1) sequester BH3-only proteins and prevent them from engaging Bak/Bax. Differences in amino acid composition within their respective $\mathrm{BH}$ domains define their specificity of binding partners, and much of this has been worked out experimentally [3]. It is these interactions among the $\mathrm{BH}$ proteins, dictated by their stoichiometry and relative affinities, which are a critical determinant of apoptosis sensitivity for a cell.

Intuitively one recognizes that a cell with excess pro-survival $\mathrm{BH}$ proteins (such as Bc12) will have a survival advantage. Perhaps less intuitively, this state may also render a cancer cell particularly vulnerable to additional stressors. Not all BH3-only proteins are equally capable of triggering Bak/ Bax activation, but Bim and Bid are uniquely adept. Cancer cells encountering stressors that activate Bid or Bim may survive if sufficient $\mathrm{Bcl} 2$ proteins are available to neutralize them. However, this state whereby activated Bid or $\mathrm{Bim}$ is bound to $\mathrm{Bcl} 2$ primes the cells such that an additional $\mathrm{BH} 3$-only signal (e.g., one activated by chemotherapy) can displace them leading to cell death. Indeed, this can be experimentally demonstrated [3, 4].

An appreciation of these pathways has led to the development of therapeutics termed BH3 mimetics (or Bcl2 antagonists; reviewed in [5]). These small molecules antagonize pro-survival BH proteins by competitively binding within their hydrophobic pocket. They tip the balance in BH3-only signal transduction toward the activation of Bak/ Bax, rather than sequestration within Bcl2. Pre-clinical models have shown activity for this therapeutic class for cancer, and a drug from Abbott, ABT263 , is now advancing through clinical trials. ABT-263 (and its parenteral relative, ABT-737) are potent antagonists of $\mathrm{Bcl} 2, \mathrm{BclX}_{\mathrm{L}}$ and $\mathrm{Bclw}$, but have little affinity for the remaining family members, including Mcl1 [6]. This inability to antagonize Mcl1 is ABT-263's Achilles' Heel as Mcl1 expression correlates with resistance to this drug [7].

Unfortunately, none of the $\mathrm{BH} 3 \mathrm{mi}-$ metics under development are potent Mcl1 antagonists. Indeed, many "panBcl2 inhibitors" suffer from a lack of specificity (e.g., they are capable of killing cells that are Bax/Bak or Caspase 9 null), or are simply too weak to compete with native high-affinity $\mathrm{BH} 3$-only proteins for pro-survival $\mathrm{BH}$ pockets. It is for these reasons that the report by Schwickart et al. is so welcome [8], as it may provide an alternative target for Mcl1 antagonism. Mcl1's own Achilles' Heel may lie in its tight regulation and extremely short half-life. Mcl1's protein structure is unique among $\mathrm{BH}$ proteins for its extended amino-terminus and inclusion of two PEST domains characteristic of short-lived proteins. Posttranslational modification (PTM) via phosphorylation downstream of varied signaling pathways, further regulates its stability and activity, as does the ubiquitin-proteasome pathway [9].

Mcl1 is targeted for proteasomemediated degradation by the Hect E3-ligase HUWE1 (also called Mule, LASU1, ARF-BP1), accounting for its rapid turnover at steady state [10]. This E3-ligase is impeded when Mcl1 is bound to BH3-only proteins as they share carboxy-terminus binding regions and $\mathrm{BH} 3$-only proteins have higher affinity. An additional E3-ligase, $\beta$ - TrCP $(B T R C)$, operates in a phospho-dependent manner (P-Ser159) downstream of GSK3 $\beta$ to also destabilize Mcl1 [11]. Augmenting either pathway can reduce Mcl1 levels, however, pharmacologically activating physiologic functions is more difficult than inhibiting them. To date, no Mcl1 reduction strategy has capitalized on promoting Mcl1 ubiquitination. Schwickart et al. have taken an alternative approach focusing on the other half of the equation (Figure 1 , box).

Ubiquitin modification is reversible, with nearly 100 cysteine protease deubiquitinating enzymes (DUBs) identified [12]. The authors used a Flag-tagged Mcl1 and co-immunoprecipitated proteins in a DUB search that yielded USP9X. Though the USP9X LC/MSMS "hit" consisted of a single polypeptide, follow-up studies convincingly demonstrate its role in Mcll regulation. The Usp9x-Mcl1 interaction was weaker than BH3-Mcl1 interaction and involved amino-terminal domains rather than the carboxy-terminal $\mathrm{BH}$ domains. Interactions are independent of phosphorylation at Thr92 or Thr163 downstream of ERK, which also stablilizes Mcl1. Conversely, phosphorylation at Ser155, Ser159 and Thr163 downstream of GSK3 $\beta$ interferes with Usp9x interactions such that the protein is destabilized.

Importantly, the authors correlate Usp9x and Mcl1 protein levels in tumors, hypothesizing that if this DUB is a principal regulator of $\mathrm{Mcl1}$, their levels should be directly correlated. Indeed, that was the case in relatively small numbers of tumors from different cancer subtypes. Not surprisingly, the tumors most convincingly shown include follicular lymphoma, diffuse large B-cell lymphoma and multiple myeloma, all hematolymphoid tumors. It remains to be seen how extensively this association holds up within diverse solid tumors, yet in vitro assays with solid tumor cell lines support that $U S P 9 X$ plays a regulatory role here as well. The translational relevance for this discovery was assessed in vitro and in vivo. Here, shRNA silencing of USP9X less efficiently reduced Mcl1 levels than Mcl1 silencing but both augmented sensitivity to ABT-737 suggesting Mcl1 stability provided a survival bias that could be overcome. In vivo, doxycycline-regulated shRNA USP9X silencing potentiated ABT-737 activity with inhibition of tumor growth 
in the single cell line tested.

DUBs have been demonstrated to antagonize specific oncogenic and tumor suppressive E3-ligases and are viewed as emerging targets for cancer therapeutics [12]. To this list is added USP9X for its role in deubiquitinating and stabilizing Mcl1, a bona fide oncogene. The biochemical evidence for this activity is clear, but whether a therapeutic advantage is gained will require further efforts. Clearly, the Mcl1 knock-down achieved with genetic silencing is suboptimal, and even with sensitization to ABT-737, the concentration required for cytotoxicity is exceedingly high (IC50s $>10 \mu \mathrm{M})$. In contrast, the most ABT737 sensitive cells lacking Mcl1 have nearly 3-log greater sensitivity in vitro and tumor regression (rather than inhibition) can be demonstrated in vivo [6, 13]. The finding does, however, provide a rational strategy for high throughput screening approaches to identify small molecules that inhibit Usp9x-Mcl1 interaction, with a goal of potentiating
Mcl1 antagonism further.

\section{References}

1 Youle RJ, Strasser A. The BCL-2 protein family: opposing activities that mediate cell death. Nat Rev Mol Cell Biol 2008; 9:47-59.

2 Reed JC. Apoptosis-targeted therapies for cancer. Cancer Cell 2003; 3:17-22.

3 Kim H, Rafiuddin-Shah M, Tu HC, et al. Hierarchical regulation of mitochondrion-dependent apoptosis by BCL-2 subfamilies. Nat Cell Biol 2006; 8:1348-1358.

4 Certo M, Del Gaizo Moore V, Nishino $\mathrm{M}$, et al. Mitochondria primed by death signals determine cellular addiction to antiapoptotic BCL-2 family members. Cancer Cell 2006; 9:351-365.

5 Lessene G., Czabotar PE, Colman PM. BCL-2 family antagonists for cancer therapy. Nat Rev Drug Discov 2008; 7:989-1000.

6 Oltersdorf T, Elmore SW, Shoemaker $\mathrm{AR}$, et al. An inhibitor of Bcl-2 family proteins induces regression of solid tumours. Nature 2005; 435:677-681.

7 Konopleva M, Contractor R, Tsao T, et al. Mechanisms of apoptosis sensitivity and resistance to the $\mathrm{BH} 3$ mimetic ABT-737 in acute myeloid leukemia. Cancer Cell 2006; 10: 375-388.

8 Schwickart M, Huang X, Lill JR, et al. Deubiquitinase USP9X stabilizes MCL1 and promotes tumour cell survival. Nature 2009; 463:103-107.

9 Warr MR, Shore GC. Unique biology of Mcl-1: therapeutic opportunities in cancer. Curr Mol Med 2008; 8:138-147.

10 Zhong Q, Gao W, Du F, Wang X. Mule/ ARF-BP1, a BH3-only E3 ubiquitin ligase, catalyzes the polyubiquitination of Mcl-1 and regulates apoptosis. Cell 2005; 121:1085-1095.

11 Ding Q, He X, Hsu JM, et al. Degradation of Mcl-1 by beta-TrCP mediates glycogen synthase kinase 3-induced tumor suppression and chemosensitization. Mol Cell Biol 2007; 27:40064017.

12 Hoeller D, Dikic I. Targeting the ubiquitin system in cancer therapy. Nature 2009; 458:438-444.

13 Vogler M, Dinsdale D, Dyer MJ, Cohen GM. Bcl-2 inhibitors: small molecules with a big impact on cancer therapy. Cell Death Differ 2009; 16:360-367. 